\section{SM Analytical and Bioanalytical Techniques}

Article Information

Received date: May 22, 2017

Accepted date: Jun 06, 2017

Published date: Jun 09, 2017

*Corresponding author

Lian Chen, CMIC, Inc. 2860 Forbs

Avenue, Hoffman Estates, IL, 60192,

United States, Tel: +847-645-0407

(Ext 139); Fax: 847-645-0412;

Email: lian-chen@cmicgroup.com

Distributed under Creative Commons CC-BY 4.0

\section{Method Development and Validation for the Determination of Pravastatin in Human Plasma by Lc-Ms/Ms}

\author{
Lian Chen*, Paresh Joshi, Andrii Piatkivskyi, Kalem Aguilar and Jenny Lin \\ Department of Pharmaceuticals, CMIC Inc, United States
}

\section{Abstract}

A simple, rapid, sensitive and selective liquid chromatography/tandem mass spectrometry (LC/MS/MS) method was developed and validated for the quantification of pravastatin in human plasma. Pravastatin- $\mathrm{D}_{3}$ was used as an internal standard. The analyte was extracted from human plasma samples by liquid-liquid extraction technique. Due to the presence of isobaric metabolites, $3 \alpha$-iso-pravastatin and 6-epi-pravastatin, chromatographic conditions were optimized, with a C18 column by using a mixture of $0.1 \%$ acetic acid in water and acetonitrile/ methanol $(43: 57, \mathrm{v} / \mathrm{v})$ as the mobile phase at a flow rate of $0.6 \mathrm{~mL} / \mathrm{min}$. The calibration curve obtained was linear $\left(r^{2} \geq 0.9900\right)$ over the concentration range of $0.500-500 \mathrm{ng} / \mathrm{mL}$. Method validation was performed as per FDA guidelines and the results met the acceptance criteria. The method was suitable for supporting clinical studies.

\section{Introduction}

Pravastatin, hexahydro-6-hydroxy-2-methyl-8-(2-methylbutyryloxy)-1-naphthyl-3,5dihydroxyheptanoate (Figure 1), a competitive inhibitor of 3-Hydroxy-3-Methylglutaryl Coenzyme A (HMG-CoA) reductase, the rate limiting enzyme in cholesterol biosynthesis and is widely used to treat hypercholesterolemia [1]. Pravastatin is characterized as one of the best, due to the hydroxyl group attached to its decalin ring, which results in a greater hydrophilicity than other HMG-CoA reductase inhibitors [1-3].

Many in vivo and in vitro human and animal studies suggest that active transport mechanisms are involved in the pharmacokinetics of pravastatin. Major metabolites of pravastatin include the 3 -alpha-isomer of pravastatin and a glutathione conjugate. Significant human metabolites, $3 \alpha$-isopravstatin and 6-epi-pravastatin (Figure 1), may form from a nonenzymatic-acid-catalyzed reaction in the stomach. At least 15 other minor metabolites of pravastatin have been detected [4].

Numerous methods have been developed and validated for detection/estimation of Pravastatin in plasma including high performance liquid chromatography with UV detection, LC/MS/MS [514]. In addition to pravastatin, $3 \alpha$-iso-pravastatin and 6-epi-pravastatin could also be quantified in some assays $[15,16]$. In this project we attempted to combine previous experience and expertise to create reliable, sensitive, high-throughput LC-MS/MS method to determine pravastatin in human plasma by separating the major isobaric metabolites.

\section{Materials and Methods}

\section{Chemicals and reagents}

Pravastatin Sodium reference standard was obtained from U.S. Pharmacopeial Convention (Rockville, MD, USA). Pravastatin- $\mathrm{D}_{3}$ sodium salt was used as an Internal Standard (IS) and obtained from Toronto Research Chemicals (Toronto, ON, Canada). Chemical structures are presented in Figure 1. Water used for the LC-MS/MS analysis was produced by in-house ultrapure water system from Millipore (Billerica, MA, USA). Acetonitrile and methanol were of HPLC grade and purchased from Honeywell (Muskegon, MI, USA). Ammoniumformate, formic acid, tert-Butyl methyl ether (MTBE) and acetic acid were analytical grade. The blank human plasma and whole blood were purchased from BioreclamationIVT (Westbury, NY USA).

\section{Preparation of standard solutions and quality control samples}

Stock solutions of Pravastatin and Pravastatin- $\mathrm{D}_{3}$ (IS) were prepared in methanol/water (50:50, $\mathrm{v} / \mathrm{v}$ ) at a concentration of $100 \mu \mathrm{g} / \mathrm{mL}$ and stored at $5^{\circ} \mathrm{C}$. Working standard solutions of Pravastatin and IS were prepared by dilution of stock solutions in methanol/water (50:50, v/v) and stored at $5^{\circ} \mathrm{C}$. The working solutions of Pravastatin were diluted by methanol/water $(50: 50, \mathrm{v} / \mathrm{v})$ before preparations of $25,50,125,250,1250,2500,12500,22500$ and $25000 \mathrm{ng} / \mathrm{mL}$. The plasma calibration curves were prepared at concentrations of $0.500,1.00,2.50,5.00,25.0,50.0,250,450$ and $500 \mathrm{ng} / \mathrm{mL}$ by spiking $490 \mu \mathrm{L}$ of blank plasma with $10 \mu \mathrm{L}$ of working solution at appropriate concentration (to make up to $500 \mu \mathrm{L}$ total sample volume). 
The Quality Control (QC) samples were independently prepared in blank plasma at four levels of concentrations for Pravastatin: 0.500 $\mathrm{ng} / \mathrm{mL}$ (limit of quantification), $1.500 \mathrm{ng} / \mathrm{mL}$ (low concentration QC, LQC), $200 \mathrm{ng} / \mathrm{mL}$ (medium concentration QC, MQC), and $400 \mathrm{ng} /$ $\mathrm{mL}$ (high concentration QC, HQC).

\section{Sample preparation}

The analyte (Pravastatin) and its internal standard (Pravastatin- $\mathrm{D}_{3}$ ) were extracted from human plasma $(50 \mu \mathrm{L})$ by a liquid liquid extraction procedure. To the matrix blank sample and selectivity samples, $50 \mu \mathrm{L}$ of methanol/ water $(50: 50$, v/v) was added. To the zero sample, calibration standards, QC samples and analytical samples, $50 \mu \mathrm{L}$ of the IS solution $(100 \mathrm{ng} / \mathrm{mL})$ were added. To the samples, $200 \mu \mathrm{L}$ of the $0.2 \mathrm{M}$ ammonium formate (pH 3.6) and $3 \mathrm{~mL}$ of Tert-Butyl methyl ether were added. The samples were mixed for approximately 5 minute with a vortex mixer after seal followed by centrifugation (e.g. preset value: $2,000 \times \mathrm{g}, 4^{\circ} \mathrm{C}, 5$ minutes). The sample tubes were immersed in a dry ice/acetone bath (e.g. for 2 minutes) to freeze the aqueous layer. The organic layer was transferred to glass tubes and evaporated to dryness under a stream of nitrogen gas in a temperature-controlled water bath unit set at $40^{\circ} \mathrm{C}$, approx. 15 minutes. The residue was reconstituted in $200 \mu \mathrm{L}$ of $0.1 \%$ acetic acid in water/ [acetonitrile/methanol $(43: 57, \mathrm{v} / \mathrm{v})$ ] $(55: 45, \mathrm{v} / \mathrm{v})$ by mixing for approximately 30 seconds with a vortex mixer after seal. The reconstituted samples were centrifuged briefly using the "flash" function on an AX-320 centrifuge, and then stored refrigerated (set temperature: $5^{\circ} \mathrm{C}$ ) until analysis.

\section{LC-MS/MS instrument and conditions}

Shimadzu Nexera system (SIL-30AC, LC-30AD, DGU-20A5, CTO-20A, Rackchanger II) was used. Mass spectrometric detection was performed on an AB SciexTriple Quad 5500 instrument using MRM. Data processing was performed on Analyst 1.5.1 software package (SCIEX). Detection was performed by Turbo ionspraynegative mode with Unit Resolution. For Pravastatin, the $\mathrm{MH}-(\mathrm{m} / z$ 423.2) was monitored as the precursor ion and a fragment at $m / z 321.2$ was chosen as the product ion. For internal standard, the MH- $(m / z 426.3)$ was monitored as the precursor ion and a fragment at $m / z 321.1$ was monitored as the product ion. Mass parameters were optimized as source temperature $600^{\circ} \mathrm{C}$, nebulizer gas (GS1) $40 \mathrm{psi}$, turbo gas (GS2) 60 psi,curtain gas 30 psi, Collision gas 7 psi, ion spray (IS) voltage $-4500 \mathrm{~V}$, declustering potential $-110 \mathrm{~V}$, collision energy $-20 \mathrm{~V}$, collision cell exit potential $-8 \mathrm{~V}$ for both analyte and IS.

ACE 3 C18 $(3 \times 50 \mathrm{~mm}$, particle size $3 \mu \mathrm{m})$ was selected as the analytical column. The mobile phase composition was $0.1 \%$ acetic acid in water and acetonitrile/methanol $(43: 57, \mathrm{v} / \mathrm{v})$ in the ratio of $55: 45(\mathrm{v} / \mathrm{v})$. The flow rate of the mobile phase was set at $0.6 \mathrm{~mL} /$ $\mathrm{min}$. The column temperature was set at $40^{\circ} \mathrm{C}$. The retention time of Pravastatin and IS was found to be $2.4 \mathrm{~min}$ approximately at overall 3.5 min run time.

\section{Method validation}

A thorough validation of the method was carried out as per the US FDA guidelines [17]. The analytical method was fully validated in human plasma with respect to selectivity, matrix effects, intraand inter-assay precision and accuracy, recovery, stability in plasma (freeze/thaw, short-term, long-term) post-preparative stability (re- injection reproducibility, extract stability and batch length stability), whole blood stability, dilution integrity, Lower Limit Of Quantitation (LLOQ), carryover, stock and working solution stability.

Selectivity was demonstrated by analysis of blank plasma from a minimum of 6 individual sources $(\mathrm{n}=1 \mathrm{each}$ ) and a blank consisting of a pool $(n=1)$ of the individual sources and a pooled plasma sample $(\mathrm{n}=1)$ spiked with concomitant drugs and isomers. In at least $90 \%$ of the individual sources and each of the pooled samples, the peak area response of the interfering peaks should be less than $20 \%$ of the mean peak area in the acceptable LLOQ calibration standards for the analyte, and less than $5 \%$ of the mean peak area of the IS in the acceptable LLOQ calibration standards.

Calibration standards were freshly prepared in duplicate in plasma and both replicates were assayed to generate the calibration curve in at least 3 separate batches (on at least two different days). The linearity (correlation coefficient: $r$ ) of the calibration curves and the accuracy of the back-calculated value at each calibration standard level were evaluated. Curves for intra and inter-assay precision and accuracy were used for calibration curve evaluation. The correlation coefficient ( $r$ ) should not be less than 0.9900 . The accuracy for calibration standards should be within $\pm 15 \%$ of the nominal value at every concentration except for the LLOQ calibration standards, where it should be within $\pm 20 \%$ of the nominal value. At least $75 \%$ non-zero standards and a minimum of six calibration standard levels should meet the above criteria in each calibration standard set. Half of LLOQ and ULOQ standards should meet the above criteria for the core intra and inter-assay precision and accuracy.

For intra/inter-assay precision and accuracy, LLQC, LQC, MQC, and HQC ( $\mathrm{n}=6$ at each level) were analyzed in three batches on at least two different days. For each batch, the intra/inter-assay precision (CV) and accuracy were calculated for each concentration level, and at least five values must be available for each concentration level to calculate statistics. The CV of each batch should not exceed $15 \%$ for each QC level except for the LLQC, where the CV should not exceed $20 \%$. The accuracy of the mean concentration of each batch should be within $\pm 15 \%$ of the nominal values for each QC level except for the LLQC, where the mean value should be within $\pm 20 \%$ of the nominal value. At least $67 \%$ of QC sample accuracy for each batch should be within $\pm 15 \%$ of their respective nominal values except LLOQ where the accuracy should be within $\pm 20 \%$ of nominal value. No more than $50 \%$ of the QC replicates at the same concentration level can be outside of $\pm 15 \%$ of the nominal values except LLQC, where the accuracy can be outside of $\pm 20 \%$ of nominal value.

Matrix effects were evaluated using three types of plasma; normal plasma, 5\% hemolyzed plasma and hyperlipidemia plasma. The plasma samples were analyzed for matrix effects at the LQC and HQC concentration levels ( 6 sources for each type, $n=1$ each at each level).

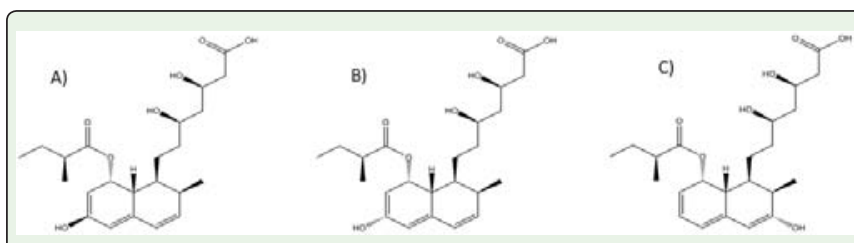

Figure 1: Structure of A) Pravastatin; B) 6-epi-Pravastatin; C) 3a-isoPravastatin. 
The CV of the IS-normalized Matrix Factor (MF) calculated from the 6 sources of each type of plasma should not exceed $15 \%$ at each concentration level.

The recovery of analyte, Pravastatin, was determined by comparing the peak area ratios (Analyte/IS) of the recovery samples at three concentration levels (LQC, MQC and HQC, $n=5$ for each level) with the mean peak area ratios of the corresponding reference samples prepared in extracted blank matrix samples $(n=5$ for each level). The CV of recovery for Pravastatin was calculated for each concentration level and should not exceed 15\%. Similarly, the recovery of IS, Pravastatin- $\mathrm{D}_{3}$, was determined by comparing the corresponding peak area ratios [1/(Analyte/IS)] of the IS recovery samples at HQC level $(n=5)$ with the mean peak area ratio of the corresponding reference samples. The CV of recovery for IS was calculated and should not exceed $15 \%$.

The concentrations of the QC samples ( $\mathrm{n}=3$ for LQC and HQC) for stability in plasma were verified immediately after preparation and then again after stability periods. The initial concentration verification can be performed during intra-assay or inter-assay precision and accuracy determination using those samples or during other validation tests. The analyte should be considered stable during a period of storage or throughout a procedure, if the mean concentration of stability samples at each level is within $\pm 15 \%$ of the nominal concentration. At least $67 \%$ (four out of six) of the batch QC samples must have accuracy within $\pm 15 \%$ of their respective nominal values.

Whole blood stability samples ([Low QC sample for whole blood stability, LQCw] and [Mid QC sample for whole blood stability, $\mathrm{MQCw}$, total $\mathrm{n}=5$ at each concentration level) were held under room temperature for 0 and 2 hours. Plasma fraction was separated from the red blood cells and then analyzed. Whole blood stability was evaluated based on the mean peak area ratio of the stored samples. The analyte (Pravastatin) should be considered stable during a period of time or throughout a procedure, if the mean peak area ratio analyte/IS of the stored samples is within $\pm 15 \%$ of the mean peak area ratio of 0 hour.

The Dilution QC (DQC) at analyte concentration level of $2000 \mathrm{ng} /$ $\mathrm{mL}(\mathrm{n}=1)$ was diluted 10 fold ( $\mathrm{n}=6 \mathrm{each})$ with blank plasma (pooled)



Figure 2: MRM chromatogram obtained from sample spiked with Pravastatin Isomers. and the diluted samples were analyzed with calibration standards and batch QC samples. The CV and accuracy were calculated based on the measured concentrations and the mean value, respectively. At least five values must be available for each level to calculate statistics. The CV should not exceed $15 \%$ and the accuracy of the mean value should be within $\pm 15 \%$ of the nominal values. At least $67 \%$ of DQC sample accuracy should be within $\pm 15 \%$ of their respective nominal values.

\section{Results}

\section{Method development}

Separation of Pravastatin and isomers was attempted using various combinations of acetonitrile, methanol and buffer with varying contents of each component on different columns like ACE, Hypersil, Waters, Zorbax, and Intertsil. It was found that a mixture of $0.1 \%$ acetic acid in water and Acetonitrile/methanol (43:57, v/v) with ACE 3 C18 column $(3 \times 50 \mathrm{~mm}$, particle size $3 \mu \mathrm{m})$ could achieve this purpose. The mass spectrum condition was optimized with infusion and Flow Injection Analysis (FIA) of the Pravastatin and IS solutions using Analyst software.

\section{Selectivity}

The presence of interfering peaks was determined in the MRM chromatograms for each selectivity sample. In at least $90 \%$ of the individual sources and each of the pooled samples, the peak area response of the interfering peaks was less than $20 \%$ of the mean peak area in the acceptable LLOQ calibration standards for the analyte, and less than $5 \%$ of the mean peak area of the IS in the acceptable LLOQ calibration standards. The results met the validation acceptance criteria.

Selectivity was also demonstrated by analysis of a pooled plasma sample $(\mathrm{n}=1)$ spiked with isomers. There was no interference peak at the eluting position of IS, but interfering peak more than $20 \%$ of the mean peak area in the acceptable LLOQ calibration standards was observed at the eluting position of Pravastatin. It was confirmed that the eluting positions of $3 \alpha$-Hydroxy Pravastatin and 6-epi-Pravastatin were well separated from Pravastatin (Figure 2).

The MRM chromatogram for the selectivity sample is presented in Figure 3.

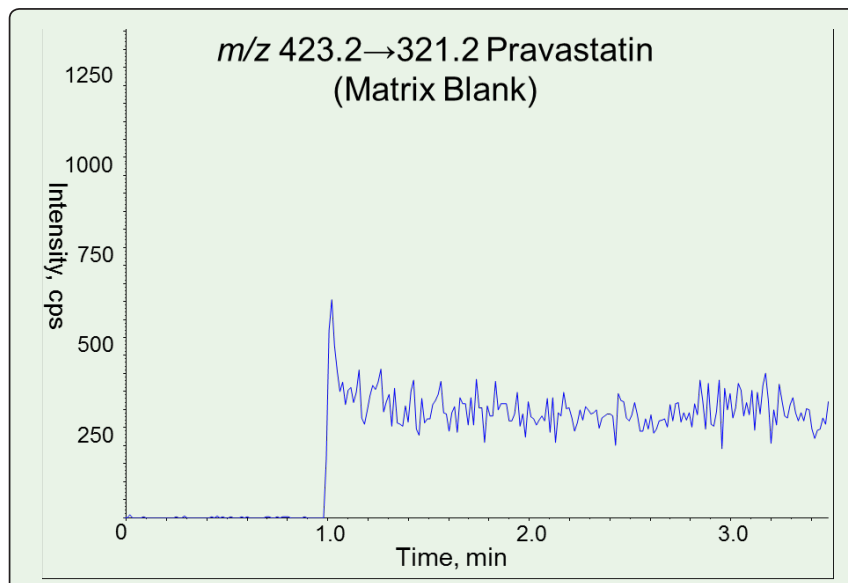

Figure 3: MRM chromatogra obtained from selectivity sample. 


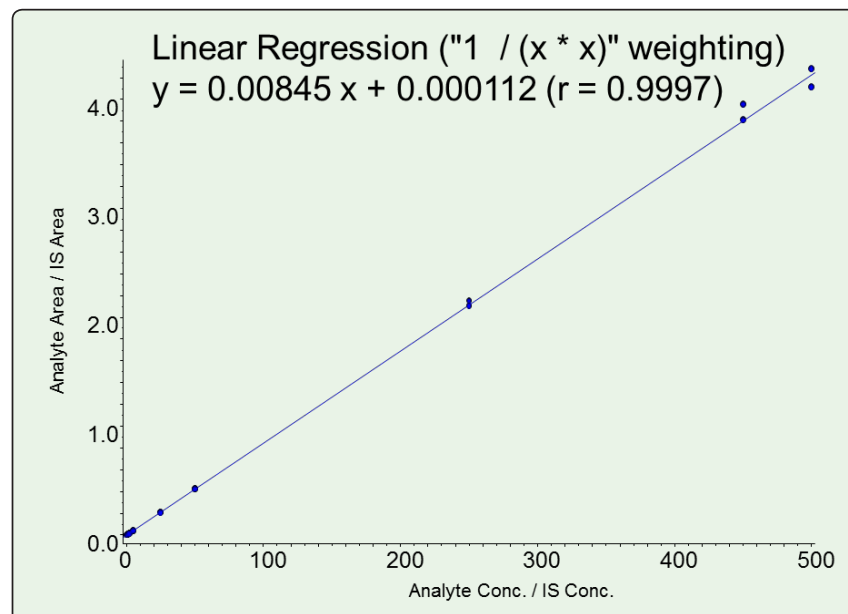

Figure 4: A Representative Fitted Calibration Curve for Pravastatin in Human Plasma.

\section{Calibration curve}

The accuracy of at least $75 \%$ of calibration standards and a minimum of six calibration standard levels in each calibration standard set were within $\pm 15 \%$ ( $\pm 20 \%$ for the LLOQ) of the nominal concentration at every concentration; therefore, calibration standards met acceptance criteria. The linearity (correlation coefficient: $r$ ) of each calibration curve met acceptance criteria, as each was $>0.9900$ (Figure 4 and Table 1). Representative MRM chromatogram for LLOQ is presented in Figure 5.

\section{Intra-assay precision and accuracy}

LLQC, LQC, MQC, and HQC ( $\mathrm{n}=6$ at each level) were evaluated in three batches on at least two different days. LLQC $(n=6)$ was analyzed in one batch. The intra-assay data met acceptance criteria, with \%CV $\leq 15 \%$ for each QC level ( $\leq 20 \%$ for LLQC) and accuracy within $\pm 15 \%$ of the nominal value for each QC level ( $\pm 20 \%$ for LLQC) and at least $67 \%$ of QC sample accuracy for each batch should be within $\pm 15 \%$ of their respective nominal values ( $\pm 20 \%$ for LLQC). The intra-assay precision and accuracy results are presented in table 2.

\section{Inter-assay precision and accuracy}

Inter-assay accuracy and precision were evaluated from the plasma quality control pool (LLQC, LQC, MQC and HQC) in 3

Table 1: Calibration Curve Data for Pravastatin in Human Plasma.
\begin{tabular}{|c|c|c|c|}
\hline $\begin{array}{c}\text { Nominal Concentration } \\
\text { (ng/mL) }\end{array}$ & $\begin{array}{c}\text { Mean observed } \\
\text { concentration } \\
\text { (ng/mL) }\end{array}$ & $\begin{array}{c}\text { CV } \\
\text { (\%) }\end{array}$ & $\begin{array}{c}\text { Accuracy } \\
\text { (\%) }\end{array}$ \\
\hline S1 (0.500) & 0.505 & 5.2 & 101.0 \\
\hline S2 (1.00) & 0.984 & 6.4 & 98.4 \\
\hline S3 (2.50) & 2.47 & 3.8 & 98.7 \\
\hline S4 (5.00) & 5.03 & 2.9 & 100.5 \\
\hline S5 (25.0) & 25.1 & 1.7 & 100.3 \\
\hline S5 (50.0) & 50.1 & 2.2 & 100.3 \\
\hline S6 (250) & 247 & 3.1 & 98.8 \\
\hline S7 (450) & 459 & 2.4 & 101.9 \\
\hline S8 (500) & 500 & 2.8 & 100.1 \\
\hline
\end{tabular}

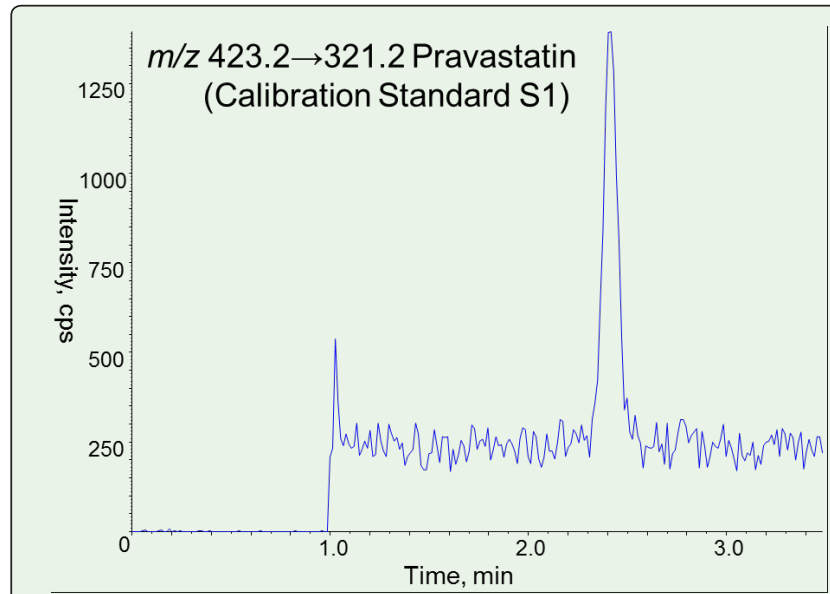

Figure 5: MRM chromatogram obtained from a LLOQ sample $(0.500 \mathrm{ng} /$ $\mathrm{mL})$.

validation runs over three separate batches on at least two different days, where each QC was analyzed $n=6$ per run. The intra-assay precision and accuracy results for human plasma were also used as the inter-assay precision and accuracy results. The inter-assay precision and accuracy data met the acceptance criteria. The overall $\%$ CV was $<15 \%$ for each QC level ( $\leq 20 \%$ for LLQC). The accuracy of the overall mean concentration was within $\pm 15 \%$ of the nominal values for each QC level ( $\pm 20 \%$ for LLQC). More than $67 \%$ of QC sample's accuracy for each batch at each level was within $\pm 15 \%$ of their respective nominal values $( \pm 20 \%$ for LLQC). The inter-assay precision and accuracy results are presented in table 2 .

\section{Matrix effects}

Matrix effects were evaluated by analysis of 6 sources ([ 3 males and 3 females], $n=1$ each at each concentration level) of blank matrix (normal plasma, 5\% hemolyzed plasma and lipemic plasma) at the LQC and HQC concentration levels. IS normalized MF was calculated for each analyte and source of matrix, this was done by calculating the ratio of the peak area ratio in the presence of matrix (measured by analyzing blank matrix spiked with analyte after extraction), to the peak area ratio in absence of matrix (pure solution of the analyte). The \%CV of the IS-normalized MF calculated from the 6 sources of matrix did not exceed $15 \%$. The results met the validation acceptance criteria.

Table 2: Intra/Inter-assay Precision and Accuracy for Pravastatin in Human Plasma.

\begin{tabular}{|c|c|c|c|c|}
\hline & $\begin{array}{c}\text { QC } \\
\text { Sample }\end{array}$ & $\begin{array}{c}\text { Mean observed } \\
\text { concentration }\end{array}$ & CV & Accuracy \\
\hline & $(\mathrm{ng} / \mathrm{mL})$ & $(n g / m L)(n=6)$ & $(\%)$ & (\%) \\
\hline \multirow{4}{*}{$\begin{array}{l}\text { Intra-assay precision and } \\
\text { accuracy }\end{array}$} & $\begin{array}{l}\text { LLQC } \\
(0.500)\end{array}$ & 0.518 & 2.7 & 103.7 \\
\hline & LQC (1.50) & 1.54 & 1.5 & 102.5 \\
\hline & MQC (200) & 200 & 4.2 & 99.8 \\
\hline & HQC (400) & 391 & 2.4 & 97.8 \\
\hline \multirow{4}{*}{$\begin{array}{l}\text { Inter-assay precision and } \\
\text { accuracy }\end{array}$} & $\begin{array}{l}\text { LLQC } \\
(0.500)\end{array}$ & 0.52 & 5.1 & 104.0 \\
\hline & LQC (1.50) & 1.51 & 3.5 & 100.6 \\
\hline & MQC (200) & 200 & 3.1 & 99.8 \\
\hline & HQC (400) & 393 & 1.9 & 98.2 \\
\hline
\end{tabular}




\section{Recovery}

The recovery of analyte was determined by comparing the peak area ratios (analyte/IS) of the recovery samples at three concentration levels (LQC, MQC and HQC, $\mathrm{n}=5$ for each level) with the mean peak area ratios of the corresponding reference samples ( $\mathrm{n}=5$ for each level). Similarly, the recovery of IS was determined by comparing the peak area ratios [1/(analyte/IS)] of the IS recovery samples at HQC level $(n=5)$ with the mean peak area ratio of the corresponding reference samples. The CV for each concentration level did not exceed $15 \%$ and the results met the acceptance criteria.

\section{Freeze/Thaw stability}

The influence of the physical processes of freezing and thawing on analyte stability in biological matrix was evaluated by analyzing plasma LQC and HQC stability samples ( $\mathrm{n}=3$ for each level) that were subjected to 5 freeze/thaw cycles prior to analysis. A freeze/thaw cycle consists of keeping the quality control samples frozen for at least twelve hours at $-80^{\circ} \mathrm{C}$ and at $-20^{\circ} \mathrm{C}$ and then completely thawing at room temperature (approximately $25^{\circ} \mathrm{C}$ ), with the initial freezing period lasting at least 24 hours. The freeze/thaw stability data met the validation acceptance criteria, with the accuracy of the mean value within $\pm 15 \%$ of the nominal value for each quality control level. The results of freeze/thaw stability are presented in Table 3 . The results indicate that the analyte is stable in matrix for 5 freeze and thaw cycles when stored at either $-20^{\circ} \mathrm{C}$ or $-80^{\circ} \mathrm{C}$.

\section{Short-term stability}

Short-term stability was evaluated by analyzing plasma stability samples at LQC and HQC levels ( $\mathrm{n}=3$ for each level) that were stored under frozen conditions $\left(-80^{\circ} \mathrm{C}\right)$ for at least 24 hours, completely thawed at room temperature (approximately $25^{\circ} \mathrm{C}$ ), and then subsequently stored at room temperature for approximately 24 hours. The short-term stability data met the validation acceptance criteria, with the accuracy of the mean value within $\pm 15 \%$ of the nominal value for each quality control level. The results of short term stability are presented in Table 3 . The results indicate that the analyte is stable in matrix stored at room temperature till 24 hours.

Table 3: Stability for Pravastatin in Human Plasma.

\begin{tabular}{|c|c|c|c|}
\hline & $\begin{array}{c}\text { Nominal } \\
\text { concentration } \\
(\mathrm{ng} / \mathrm{mL})\end{array}$ & $\begin{array}{l}\text { Mean observed } \\
\text { concentration } \\
(n g / m L)(n=3)\end{array}$ & $\begin{array}{c}\text { Accuracy } \\
\text { (\%) }\end{array}$ \\
\hline \multirow{2}{*}{$\begin{array}{c}\text { Room temperature stability } \\
\text { in plasma }(24 \mathrm{~h})\end{array}$} & LQC (1.50) & 1.48 & 98.6 \\
\hline & HQC (400) & 400 & 99.9 \\
\hline \multirow{2}{*}{ Extraction stability (72 h) } & LQC (1.50) & 1.62 & 108.1 \\
\hline & HQC (400) & 399 & 99.6 \\
\hline \multirow{2}{*}{ Re-injection stability (72 h) } & LQC (1.50) & 1.51 & 100.4 \\
\hline & HQC (400) & 392 & 98.0 \\
\hline \multirow{4}{*}{$\begin{array}{c}\text { Freeze/thaw stability in } \\
\text { plasma }\left(5 \text { cycles, }-20^{\circ} \mathrm{C} \text { and }\right. \\
\left.-80^{\circ} \mathrm{C}\right)\end{array}$} & LQC (1.50) & 1.62 & 108.3 \\
\hline & HQC (400) & 393 & 98.3 \\
\hline & LQC (1.50) & 1.53 & 101.8 \\
\hline & HQC (400) & 387 & 96.8 \\
\hline \multirow{4}{*}{$\begin{array}{l}\text { Long term stability in plasma } \\
\left(178 \text { days, }-20^{\circ} \mathrm{C} \text { and }-80^{\circ} \mathrm{C}\right)\end{array}$} & LQC (1.50) & 1.47 & 98.0 \\
\hline & HQC (400) & 400 & 99.9 \\
\hline & LQC (1.50) & 1.52 & 101.5 \\
\hline & HQC (400) & 393 & 98.3 \\
\hline
\end{tabular}

\section{Long-term Stability}

Long-term stability was evaluated by analyzing plasma stability samples at LQC and HQC levels ( $\mathrm{n}=3$ for each level) that were stored under frozen conditions $\left(-20^{\circ} \mathrm{C}\right.$ and $\left.-80^{\circ} \mathrm{C}\right)$ for 6 months and then processed and analyzed for long-term stability. The long-term stability data met the validation acceptance criteria, with the accuracy of the mean value within $\pm 15 \%$ of the nominal value for each quality control level. The results of long-term stability are presented in Table 3 . The results indicate that the analyte is stable in matrix when stored in a freezer set at $-20^{\circ} \mathrm{C}$ (acceptable temperature: $-25^{\circ} \mathrm{C}$ to $-15^{\circ} \mathrm{C}$ ), and in another freezer set at $-80^{\circ} \mathrm{C}$ (acceptable temperature: $-90^{\circ} \mathrm{C}$ to $-70^{\circ} \mathrm{C}$ ) till 178 days.

\section{Batch length stability}

Batch length stability was demonstrated by analyzing a run containing MB, Z, calibration standards, and LQC, MQC and HQC samples ( $\mathrm{n}=1$ at each level), with the number of expected samples represented by injecting $\mathrm{MB}$ samples or additional QC replicates, and a second set of $\mathrm{QC}$ samples, $\mathrm{MB}, \mathrm{Z}$ and calibration standards. A series of $\mathrm{MB}$ samples and additional QC replicates were injected to mimic the anticipated maximum number of unknown samples to be analyzed in one sample analysis batch. The batch length stability results indicate that maximum 120 samples can be analyzed in one run without any effect on stability.

\section{Re-injection stability}

Re-injection stability was demonstrated by re-injecting the previously injected LQC and HQC intra-assay reproducibility samples ( $\mathrm{n}=6$ for each level), as well as the corresponding previously injected calibration standards, after storage in the sample compartment refrigerator (set at $5^{\circ} \mathrm{C}$ ) for at least 72 hours after the initial injection. The results met the validation acceptance criteria for all calibration standard levels. The re-injection reproducibility results met the validation acceptance criteria, with $\% \mathrm{CV} \leq 15 \%$ and the accuracy of the mean value within $\pm 15 \%$ of the nominal value for each QC level. The results of re-injection reproducibility are presented in Table 3. These results indicate that the analyte in processed and previously injected samples is stable for at least 72 hours in an auto sampler set at $5^{\circ} \mathrm{C}$.

\section{Extract stability}

Extract stability was demonstrated by extracting LQC and HQCstability samples ( $\mathrm{n}=3$ for each level) and analyzing them after being stored in the auto sampler (set at $5^{\circ} \mathrm{C}$ ) at least 72 hours and analyzed with freshly prepared calibration standards. The extract stability results met the validation acceptance criteria, with \%CV $\leq 15 \%$ and the accuracy of the mean value within $\pm 15 \%$ of the nominal value for each QC level. The results of extract stability are presented in Table 3. Theresults indicate that the analyte in processed samples is stable till 72 hours in an autosampler set at $5{ }^{\circ} \mathrm{C}$.

\section{Stock and standard solution stability}

Stability under room temperature and frozen conditions for stock and standard solutions were evaluated, and were based on the mean peak area ratios and mean IS peak area ratios [1/(analytePravastatin/ IS)] obtained. 
Table 4: Stock Solution and Working Solution Stability of Pravastatin.

\begin{tabular}{|c|c|c|c|}
\hline & $\begin{array}{c}\text { Nominal } \\
\text { concentration }\end{array}$ & Period & $\begin{array}{l}\text { Remaining } \\
(\%)(n=3)\end{array}$ \\
\hline $\begin{array}{l}\text { Stock solutionroom temperature } \\
\text { stability }\end{array}$ & $100 \mu \mathrm{g} / \mathrm{mL}$ & 24 hours & 100.7 \\
\hline Stock solution stability at $5^{\circ} \mathrm{C}$ & $100 \mu \mathrm{g} / \mathrm{mL}$ & $\begin{array}{l}184 \\
\text { days }\end{array}$ & 100.4 \\
\hline $\begin{array}{l}\text { IS Stock solutionroom temperature } \\
\text { stability }\end{array}$ & $100 \mu \mathrm{g} / \mathrm{mL}$ & 24 hours & 95.8 \\
\hline IS Stock solution stability at $5^{\circ} \mathrm{C}$ & $100 \mu \mathrm{g} / \mathrm{mL}$ & $\begin{array}{l}184 \\
\text { days }\end{array}$ & 89.6 \\
\hline $\begin{array}{l}\text { Working solutionroom temperature } \\
\text { stability }\end{array}$ & $25 \mathrm{ng} / \mathrm{mL}$ & 24 hours & 98.4 \\
\hline Working solution stability at $5^{\circ} \mathrm{C}$ & $25 \mathrm{ng} / \mathrm{mL}$ & $\begin{array}{l}184 \\
\text { days }\end{array}$ & 100.6 \\
\hline $\begin{array}{l}\text { IS solutionroom temperature } \\
\text { stability }\end{array}$ & $100 \mathrm{ng} / \mathrm{mL}$ & 24 hours & 97.8 \\
\hline IS solution stability at $5^{\circ} \mathrm{C}$ & $100 \mathrm{ng} / \mathrm{mL}$ & $\begin{array}{c}184 \\
\text { days }\end{array}$ & 93.7 \\
\hline
\end{tabular}

Pravastatin and IS stock solutions and working solutions were held at room temperature (approximately $25^{\circ} \mathrm{C}$ ) for approximately 24 hours, and then injection samples $(n=3)$ for stock solution and working solutions stability were prepared from the stored solutions. Injection samples $(\mathrm{n}=3)$ for stock solution and working solutions stability were prepared from freshly prepared solutions (for initial values). The stability of stock solutions and working solutions met the acceptance criteria, stability mean values were within $\pm 15 \%$ of the initial mean value and the results are presented in Table 4 . The results indicate that the stock solution and working solutions for Pravastatin and IS can be stored at room temperature for at least 24 hours.

Pravastatin and IS stock solutions and working solutions were held under frozen conditions (acceptable temperature: $2^{\circ} \mathrm{C}$ to $8^{\circ} \mathrm{C}$ ) for approximately 6 months, and then injection samples $(n=3)$ for stock solution and working solutions stability were prepared from the stored solutions. Injection samples $(n=3)$ for stock solution stability and working solutions were prepared from freshly prepared solutions (for initial values). The stability of stock solutions and working solutions met the acceptance criteria, stability mean values were within $\pm 15 \%$ of the initial mean value and the results are presented in Table 4. The results indicatethat the stock solution and working solutionsfor Pravastatin and IS can be stored at refrigerated condition for at least 184 days.

\section{Whole blood stability}

The 2 hour whole blood stability samples met the acceptance criteria for Pravastatin, stability mean values were within $\pm 15 \%$ of the mean peak area ratio of the 0 hour sample. The results indicate that the analyte is stable in whole blood for at least 2 hours for Pravastatin.

\section{Dilution integrity}

The dilution integrity evaluation met the acceptance criteria, with $\% \mathrm{CV} \leq 15 \%$ for replicates and the accuracy within $\pm 15 \%$ of the nominal value for the mean value. The results indicate that plasma samples can be diluted with plasma without affecting precision and accuracy.

\section{Determination of LLOQ}

The Lower Limit of Quantitation (LLOQ) is the lowest non-zero human plasma quality control pool concentration level which can be accurately and reproducibly quantified. The LLQC was analyzed $(n=6)$ and the data met acceptance criteria, with $\% \mathrm{CV} \leq 20 \%$ and the mean accuracy was within $\pm 20 \%$ of the nominal value and at least $67 \%$ of the QC samples were within $\pm 20 \%$ of the nominal value. In addition, the analyte peak area response at the LLOQ was at least five times the response compared to the first $\mathrm{MB}$ peak response and the signal to noise ratio was at least 5 . For this validation, the LLOQ was determined to be $0.500 \mathrm{ng} / \mathrm{mL}$.

\section{Carryover}

The potential for analyte carryover from high-level to low-level samples in an injection sequence was evaluated by injecting duplicate extracted matrix blanks immediately after the ULOQ calibration standards in an intra-assay precision and accuracy validation run. Carryover exceeding acceptance criteria was not observed, as the carryover peak area was less than $20 \%$ of the peak area of Pravastatin at LLOQ and no greater than 5\% of the peak area of IS on the MRM chromatograms in the first matrix blanks injected immediately after the ULOQ calibration standards. The carryover results met acceptance criteria.

\section{Batch acceptance}

Stability analysis in matrix (except for whole blood stability and re-injection stability) and Dilution integrity were performed with freshly prepared calibration curves and freshly prepared batch QC samples ( $\mathrm{n}=2$ at LQC, MQC and HQC) unless intra- or inter-assay precision and accuracy QC samples were used as batch QC samples. At least $67 \%$ of accuracy for QC samples ( $\mathrm{n}=2$ each, total 6) for stability evaluation run were within $\pm 15 \%$ of their respective nominal values. No more than $50 \%$ of QC samples for batch acceptance at the same concentration were outside $\pm 15 \%$ of the nominal values.

\section{Discussion}

In the present investigation, we have developed a method with liquid-liquid extraction technique. The following are the advantages of the proposed method over those reported earlier: (1) Because of the use of less plasma volume $(50 \mu \mathrm{L})$, the volume of the sample to be collected per time point from an individual during the study is reduced significantly; (2) Greater sensitivity is achieved even with low plasma volumes and the method is well suited for pharmacokinetic analysis; (3) Separation of Pravastatin from isobaric metabolites excluded the inference from the plasma samples.

\section{Conclusion}

In summary, the method is described for the quantification of pravastatin from human plasma by LC-MS/MS in negative ionization mode using multiple reaction monitoring. The current method has shown acceptable precision and adequate sensitivity for the quantification of pravastatin in human plasma samples obtained for pharmacokinetic studies. The method described is simple, rapid, sensitive, selective and fully validated according to commonly accepted criteria. The cost-effectiveness and simplicity of the assay make it an attractive procedure in high-throughput bioanalysis of pravastatin.

\section{References}

1. Hatanaka T. Clinical pharmacokinetics of pravastatin: mechanisms of pharmacokinetic events. Clin Pharmacokinet. 2000; 39: 397-412. 
2. Lennernäs H, Fager G. Pharmacodynamics and pharmacokinetics of the HMG-CoA reductase inhibitors. Similarities and differences. ClinPharmacokinet. 1997; 32: 403-425.

3. Haria M, McTavish D. Pravastatin. A reappraisal of its pharmacological properties and clinical effectiveness in the management of coronary heart disease.Drugs. 1997; 53: 299-336.

4. Wehr, A. Pravastatin. Handbook of Metabolic Pathways of Xenobiotics. 2014; 2: $1-6$.

5. Otter K, Mignat C. Determination of pravastatin in human plasma by highperformance liquid chromatography with ultraviolet detection. J Chromatogr B Biomed Sci Appl. 1998; 708: 235-41

6. Bauer S,Mwinyia J, Stoecklea A, Gerloffa T, Roots I. Quantification of pravastatin in human plasma and urine after solid phase extraction using high performance liquid chromatography with ultraviolet detection. J Chromatogr B AnalytTechnol Biomed Life Sci.2005; 818 : 257-262.

7. Önal A,Sagirli O. Development of a Selective LC Method for the Determination of Pravastatin Sodium.Chromatogrhaphia. 2006; 64: 3-4.

8. Sparidans RW,lusuf D, Schinkel AH, Schellens JH, Beijnen JH. Liquid chromatography-tandem mass spectrometric assay for pravastatin and two isomeric metabolites in mouse plasma and tissue homogenates. J Chromatogr B AnalytTechnol Biomed Life sci.2010; 878: 2751-2759.

9. Tan ZR,Ouyang DS, Zhou G, Guo D, Chen Y, et al. A simple and sensitive LC-MS/MS method for determination of pravastatin in human plasma. Chinese J New Drugs. 2008; 17: 1150-1153

10. Deng JW, Kim KB, Song IS, Shon JH, Zhou HH, et al. Determination of two HMG-CoA reductase inhibitors, pravastatin and pitavastatin, in plasma samples using liquid chromatography-tandem mass spectrometry for pharmaceutical study.Biomed Chromatogr. 2008; 22: 131-135.
11. Jain DS,Subbaiah G, Sanyal M, Jain VK, Shrivastav P. A rapid and specific approach for direct measurement of pravastatin concentration in plasma by LC-MS/MS employing solid-phase extraction.Biomed Chromatogr.2007; 21 : $67-78$.

12. Kyrklund C,Backman JT, Neuvonen M, Neuvonen PJ. Effect of rifampicin on pravastatin pharmacokinetics in healthy subjects. $\mathrm{Br} \mathrm{J}$ Clin Pharmacology.2004; 57: 181-187.

13. Kawabata K,Samata N, Urasaki Y.Quantitative determination of pravastatin and R-416, its main metabolite in human plasma, by liquid chromatographytandem mass spectrometry. J Chromatogr B AnalytTechnol Biomed Life Sci. 2005; 816: 73-79.

14. Mertensa B,Cahaya B, Klinkenberga R, Streel B.An automated method for the simultaneous determination of pravastatin, 3-hydroxy isomeric metabolite, pravalactone and fenofibric acid in human plasma by sensitive liquid chromatography combined with diode array and tandem mass spectrometry detection. J Chromatogr A. 2008; 1189: 493-502.

15. Zhang X, Vernikovskaya DI, Wang X, Nanovskaya TN, Costantine M, Hankins GD, Ahmed MS.Quantitative determination of pravastatin and its metabolite 3a-hydroxy pravastatin in plasma and urine of pregnant patients by LC-MS/ MS.BiomedChromatogr. 2016; 30: 548-54

16. Mulvana D, Jemal M, PulverSC.Quantitative determination of pravastatin and its biotransformation products in human serum by turbo ion spray LC/MS MS.J Pharm Biomed Anal. 2000; 23: 851-66

17. US DHHS, FDA, CDER. Guidance for Industry: Bioanalytical Method Validation. U.S. Department of Health and Human Services, Food and Drug Administration, Center for Drug Evaluation and Research (CDER), Center for Veterinary Medicine (CV). 2001 